\title{
Acoustics of Jet Surface Interaction Scrubbing Noise
}

\author{
Abbas Khavaran \\ Vantage Partners, LLC \\ NASA Glenn Research Center
}

$20^{\text {th }}$ AIAA/CEAS Aeroacoustics Conference

16 - 20 June 2014, Atlanta GA

Supported by:

NASA Fundamental Aeronautics Program

Fixed Wing Project 


\section{Motivation}

Interaction of jet exhaust with nearby solid surfaces:

- Hybrid Wing Body (HWB) concepts

- High aspect ratio rectangular exhaust with extended beveled surfaces

- Over the wing engine mount

- Nearby structural components could provide noise shielding

- They could also produce new sources of sound

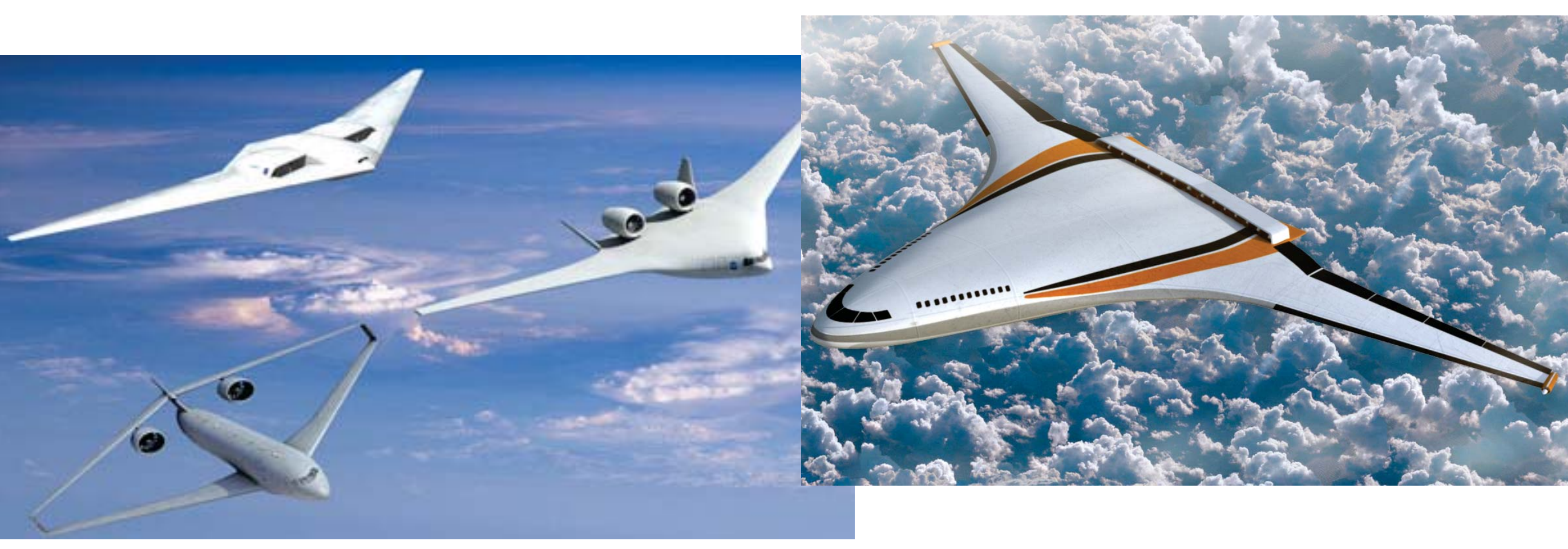




\section{Measurements* \\ Rectangular Jet ( $A R=8)$}
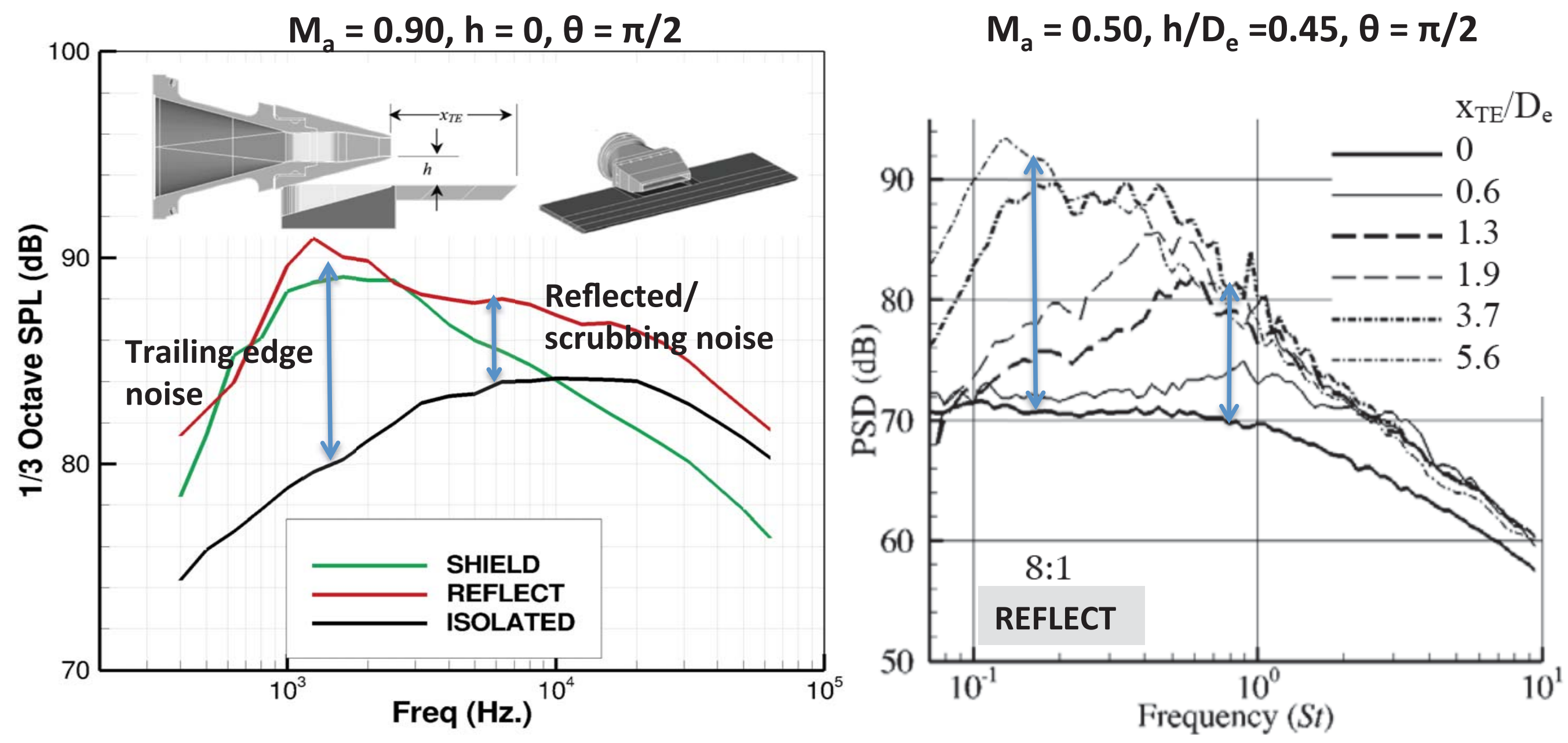

$$
\mathrm{X}_{\mathrm{TE}} / \mathrm{D}_{\mathrm{e}}=5.6, \mathrm{D}_{\mathrm{e}}=2.14^{\prime \prime}
$$

*James Bridges, AIAA-2014-0876 


\section{Outline}

- Governing Equations

- Propagation GF Applicable to High-AR Rectangular Jet

- A Parametric Study of the GF

- Frequency

- Temperature

- Source Location

- Directivity/ Flight Effect

- Wall Impedance Effect

- Reflected / Isolated Jet 


\section{Scrubbing Noise}

- NS Equations $\rightarrow$ (Mean Flow + Linear Eqs. for Fluctuations)

- Locally Parallel Mean Flow

- Compressible

- Constant Static Pressure

- Ideal Gas Law

Variable density inhomogeneous PB eq.

$$
\begin{aligned}
& L \pi^{\prime}=\Gamma, \quad \pi^{\prime} \simeq \frac{p^{\prime}(\vec{x}, t)}{\gamma \bar{p}} \\
& L \equiv D\left(D^{2}-\frac{\partial}{\partial x_{j}}\left(c^{2} \frac{\partial}{\partial x_{j}}\right)\right)+2 c^{2} \frac{\partial U}{\partial x_{j}} \frac{\partial^{2}}{\partial x_{1} \partial x_{j}}, \quad D \equiv \frac{\partial}{\partial t}+U \frac{\partial}{\partial x_{1}}
\end{aligned}
$$

- Source term $\Gamma$ is defined according to the generalized Acoustic Analogy, Goldstein 2010) 


\section{Green's Function Method}

$$
\begin{aligned}
& \pi^{\prime}(\vec{x}, t)=\int_{\vec{y}} \int_{\tau} G(\vec{x}, t ; \vec{y}, \tau) \Gamma(\vec{y}, \tau) d \tau d \vec{y} \\
& L G(\vec{x}, t ; \vec{y}, \tau)=\delta(\vec{x}-\vec{y}) \delta(t-\tau)
\end{aligned}
$$

- Wetted side of the plate only

(scattered noise component discussed by Goldstein et al, 2013)

$$
\begin{aligned}
& \text { Transform: } \begin{array}{l}
\left(x_{1}, x_{2}, t\right) \rightarrow\left(k_{1}, k_{2}, \omega\right) \\
\qquad(\vec{x}, t ; \vec{y}, \tau) \rightarrow \hat{G}\left(\vec{k}_{t}, x_{3} ; y_{3}, \omega\right) \quad \vec{k}_{t} \equiv\left(k_{1}, k_{2}\right) \\
\frac{\partial^{2} \hat{G}}{\partial x_{3}^{2}}+\left(\frac{\left(c^{2}\right)^{\prime}}{c^{2}}-\frac{2 k_{1} U^{\prime}}{-\omega+k_{1} U}\right) \frac{\partial \hat{G}}{\partial x_{3}}+\left(\frac{\left(-\omega+k_{1} U\right)^{2}}{c^{2}}-k_{1}^{2}-k_{2}^{2}\right) \hat{G}=\frac{i}{(2 \pi)^{3}} \frac{\delta\left(x_{3}-y_{3}\right)}{c^{2}\left(-\omega+k_{1} U\right)}
\end{array}
\end{aligned}
$$

- Far-field spectral density

$$
\overline{p^{2}}(\vec{x}, \omega)=\iint_{\vec{y}} \int_{\tau=-\infty}^{\infty} \mathbf{G}^{*}(\vec{x}, \vec{y}-\vec{\xi} / 2 ; \omega) \mathbf{G}(\vec{x}, \vec{y}+\vec{\xi} / 2 ; \omega) q(\vec{y}, \vec{\xi}, \tau) e^{i \omega \tau} d \tau d \vec{\xi} d \vec{y}
$$




\section{GF Method (cont'd)}

- The Eq is re-arranged into a self-adjoint $2^{\text {nd }}$ order ODE

- Two linearly independent solutions $V_{j}\left(\vec{k}_{t}, x_{3}, \omega\right), j=1,2$

$$
\begin{array}{c|c}
V_{j}^{\prime \prime}+f\left(\vec{k}_{t}, x_{3}, \omega\right) V_{j}=0 & \begin{array}{l}
V_{1}\left(x_{3}\right)=1 \\
\text { IVP }
\end{array} \\
x_{3}=0 & \begin{array}{l}
\frac{\partial V_{1}\left(x_{3}\right)}{\partial x_{3}}-\psi V_{1}\left(x_{3}\right)=0, \quad \psi\left(k_{1}, \omega, \bar{Z}\right)=\left(\frac{i \kappa_{0}}{\bar{Z}} \frac{c_{\infty}^{2}}{c^{2}(0)}+\frac{c^{\prime}(0)}{c(0)}+\frac{k_{1}}{\omega} U^{\prime}(0)\right) \\
\bar{Z}(\omega)=\frac{\left(\hat{p}^{\prime} / \hat{v}_{3}^{\prime}\right)}{\rho_{\infty} c_{\infty}}
\end{array} \\
\text { BVP } & \begin{array}{l}
V_{2}\left(x_{3}\right)=1, \quad x_{3}=0 \\
\frac{\partial V_{2}\left(x_{3}\right)}{\partial x_{3}}+i \chi_{\infty} V_{2}=0, \quad x_{3} \rightarrow \infty
\end{array}
\end{array}
$$

- Radiation condition

$$
\chi_{\infty}^{2} \equiv\left(-\kappa_{o}+k_{1} M_{\infty}\right)^{2}-k_{1}^{2}-k_{2}^{2}>0, \quad \kappa_{o}=\omega / c_{o}
$$




\section{GF Method (cont'd)}

$$
\begin{gathered}
\mathbf{G}(\vec{x}, \vec{y} ; \omega)=\frac{i}{(2 \pi)^{3}} \frac{1}{c\left(y_{3}\right) c\left(x_{3}\right)} \iint_{k_{1}} \frac{-\omega+k_{1} U\left(x_{3}\right)}{\left(-\omega+k_{1} U\left(y_{3}\right)\right)^{2}} \frac{b_{2} V_{1}\left(\vec{k}_{t}, y_{3}, \omega\right)}{W_{o}\left(\vec{k}_{t}, \omega, \bar{Z}\right)} e^{i \Theta\left(\vec{k}_{t}, \vec{x}, \omega\right)} d k_{1} d k_{2} \\
\Theta\left(\vec{k}_{t}, \vec{x}, \omega\right)=k_{1}\left(x_{1}-y_{1}\right)+k_{2}\left(x_{2}-y_{2}\right)-\chi_{\infty} x_{3} \\
V_{2}\left(\vec{k}_{t}, x_{3}, \omega\right)=b_{2}\left(\vec{k}_{t}, \omega\right) e^{-i \chi_{\infty} x_{3}} \quad x_{3} \rightarrow \infty \quad \hat{A}_{3} \quad R
\end{gathered}
$$

- Stationary Phase solution $\left(\kappa_{o} R \gg 1, \kappa_{o}=\omega / c_{\infty}\right)$

$$
\vec{k}_{t}^{s}=\kappa_{o}\left(\sin \phi^{s} \cos \theta^{s}, \cos \phi^{s}\right)
$$

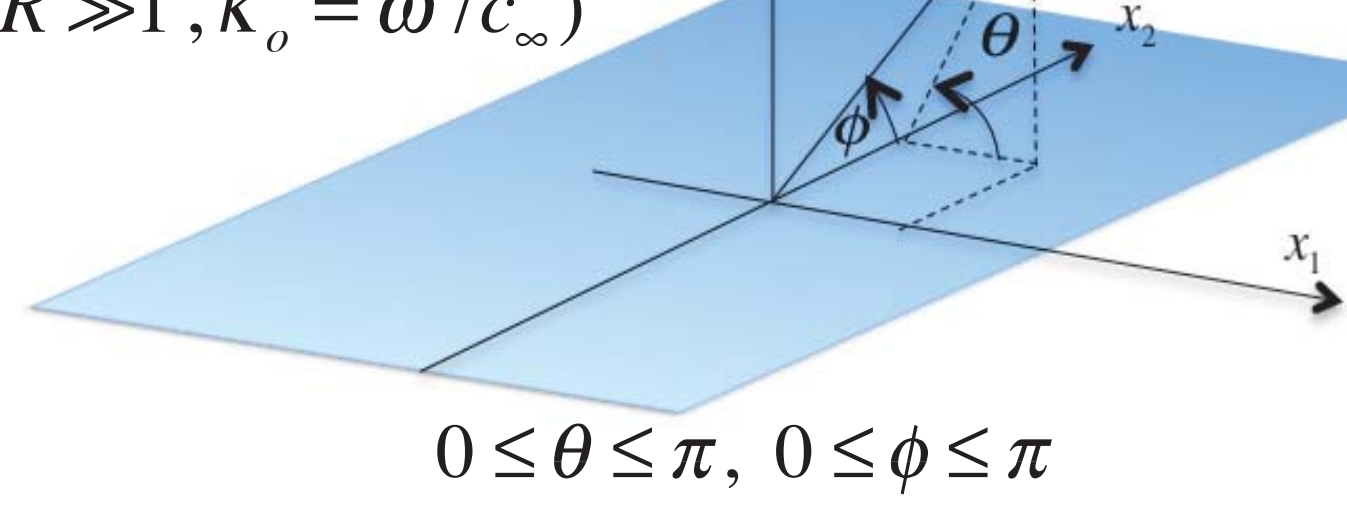

$$
\mathbf{G}(\vec{x}, \vec{y} ; \omega) \sim-i \frac{e^{i \Theta\left(\vec{k}_{t}^{s}, \vec{x}, \omega\right)}}{(2 \pi)^{3} R} \frac{\sin \theta^{s} \sin ^{2} \phi^{s}}{c_{\infty}^{2} c\left(y_{3}\right)} \frac{b_{2}\left(\vec{k}_{t}^{s}, \omega\right) V_{1}\left(\vec{k}_{t}^{s}, y_{3}, \omega\right)}{W_{o}\left(\vec{k}_{t}^{s}, \omega, \bar{Z}\right)} \frac{\mathfrak{I}}{\left(1-\frac{U\left(y_{3}\right)}{c_{\infty}} \sin \phi^{s} \cos \theta^{s}\right)^{2}}
$$




\section{Stationary-Phase Point}

- Stationary point angles are obtained from

$\tan \theta=-S\left(\theta^{s}, \phi^{s}, M_{\infty}\right) /\left(M_{\infty}+\left(1-M_{\infty}^{2}\right) \cos \theta^{s} \sin \phi^{s}\right)$

$\sin \theta \tan \phi=-S\left(\theta^{s}, \phi^{s}, M_{\infty}\right) / \cos \phi^{s}$

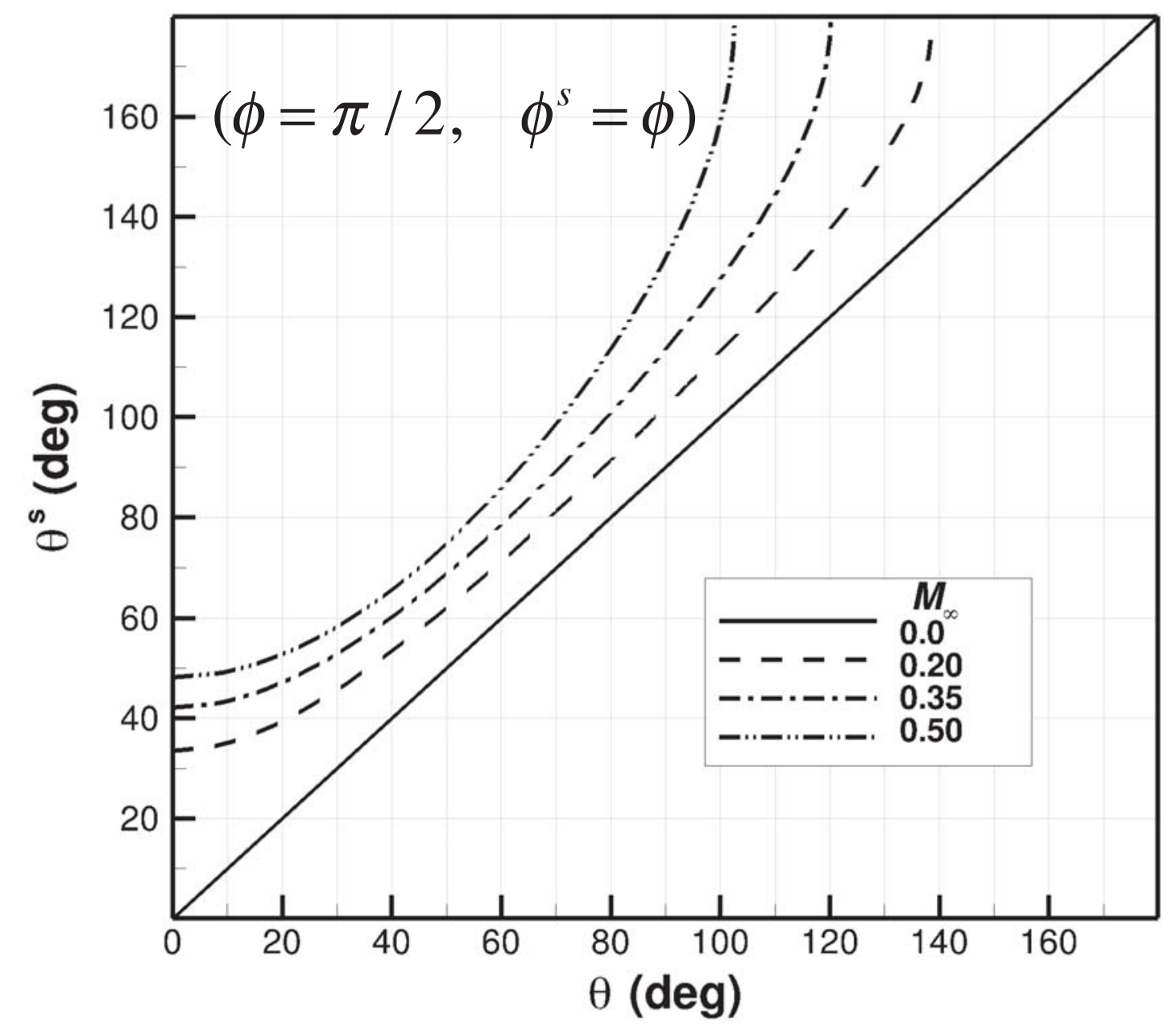




\section{Temperature \& Velocity Profiles}

$\left(M_{\infty}=0, U_{j} / c_{\infty}=0.90\right)$

- Analytical profiles are selected for mean velocity $\&$ static temperature

$$
\eta=y_{3} / D_{j}
$$
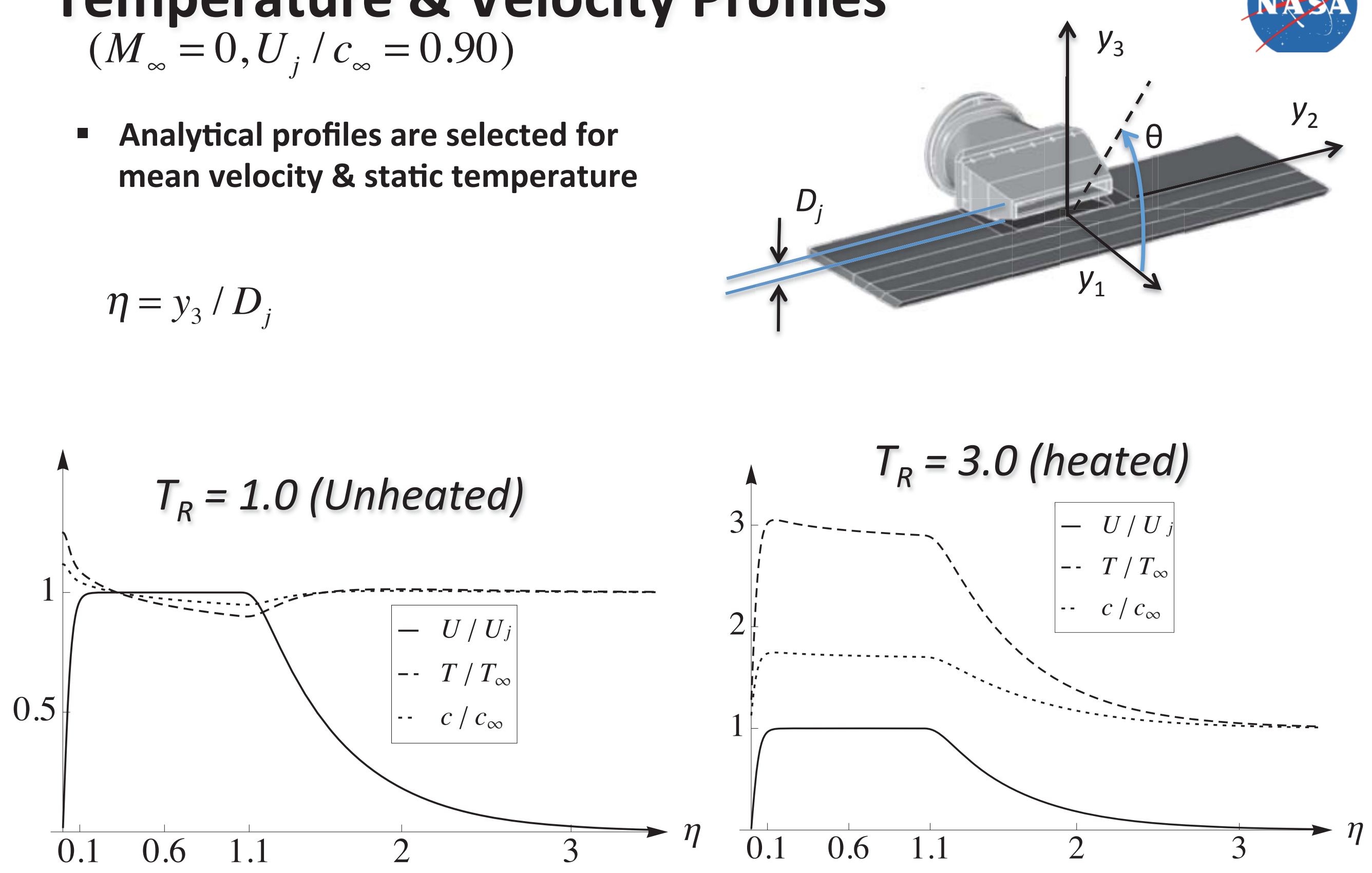
$\left(\phi=\pi / 2, \theta=\pi / 4, U_{j} / c_{\infty}=0.90, T_{R}=3.0\right)$

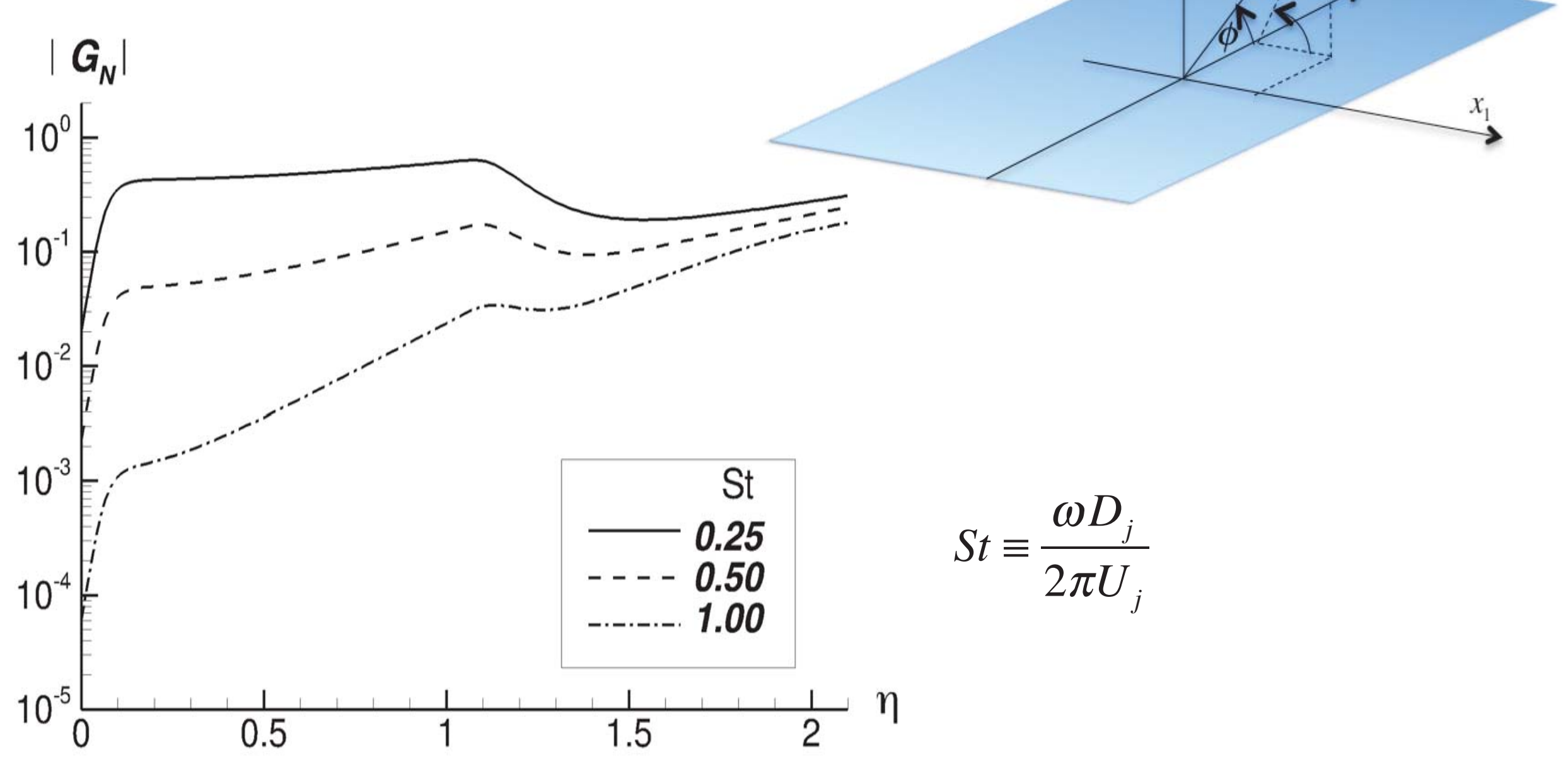

- GF amplitude decreases with increasing frequency

- In a uniform mean flow $G_{N} \sim 1 / \omega$ 


\section{Effect of Temperature}

$\left(\phi=\pi / 2, \theta=\pi / 4, S t_{o}=0.25, U_{j} / c_{\infty}=0.90\right)$

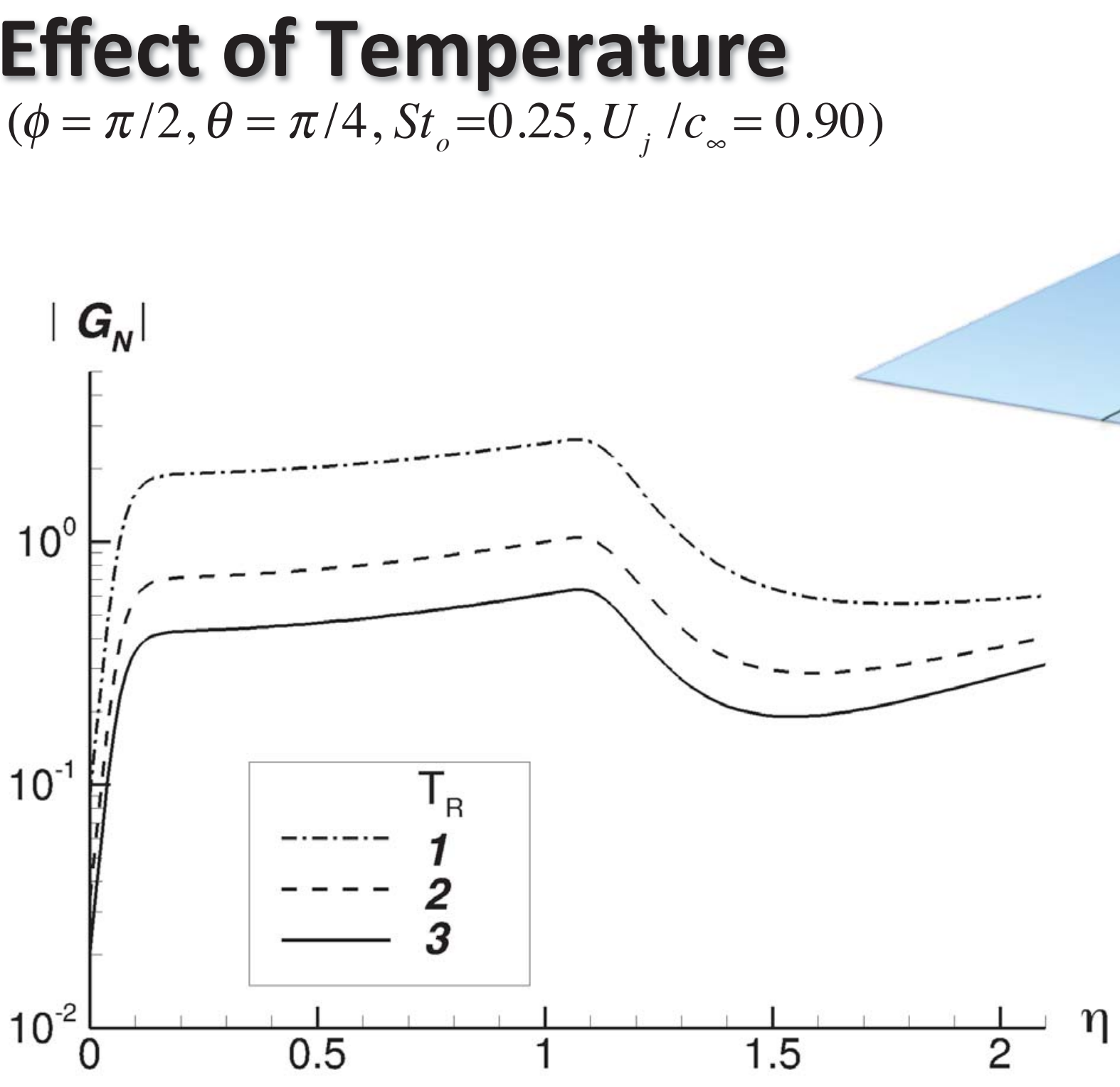

- GF amplitude decreases with increasing temperature 


\section{Effect of Surface Impedance}

$\left(\phi=\pi / 2, \theta=\pi / 4, S t_{o}=0.25, U_{j} / c_{\infty}=0.90, T_{R}=1.0\right)$

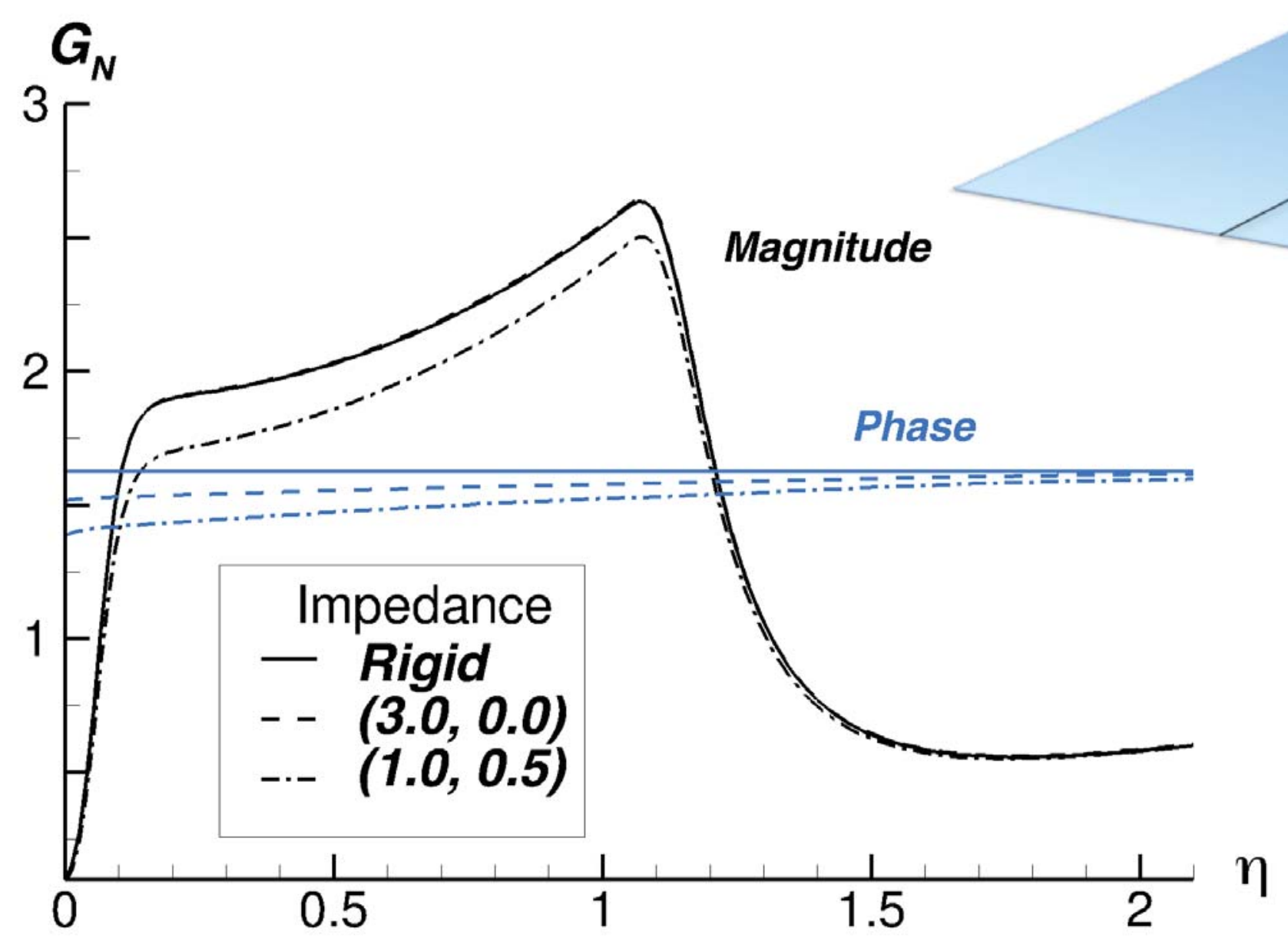

- Phase factor depends on source location for non-rigid surface 


\section{Flight Effect on Directivity}

$\left(\phi=\pi / 2, S t_{o}=0.25, U_{j} / c_{\infty}=0.90, T_{R}=1.0\right)$

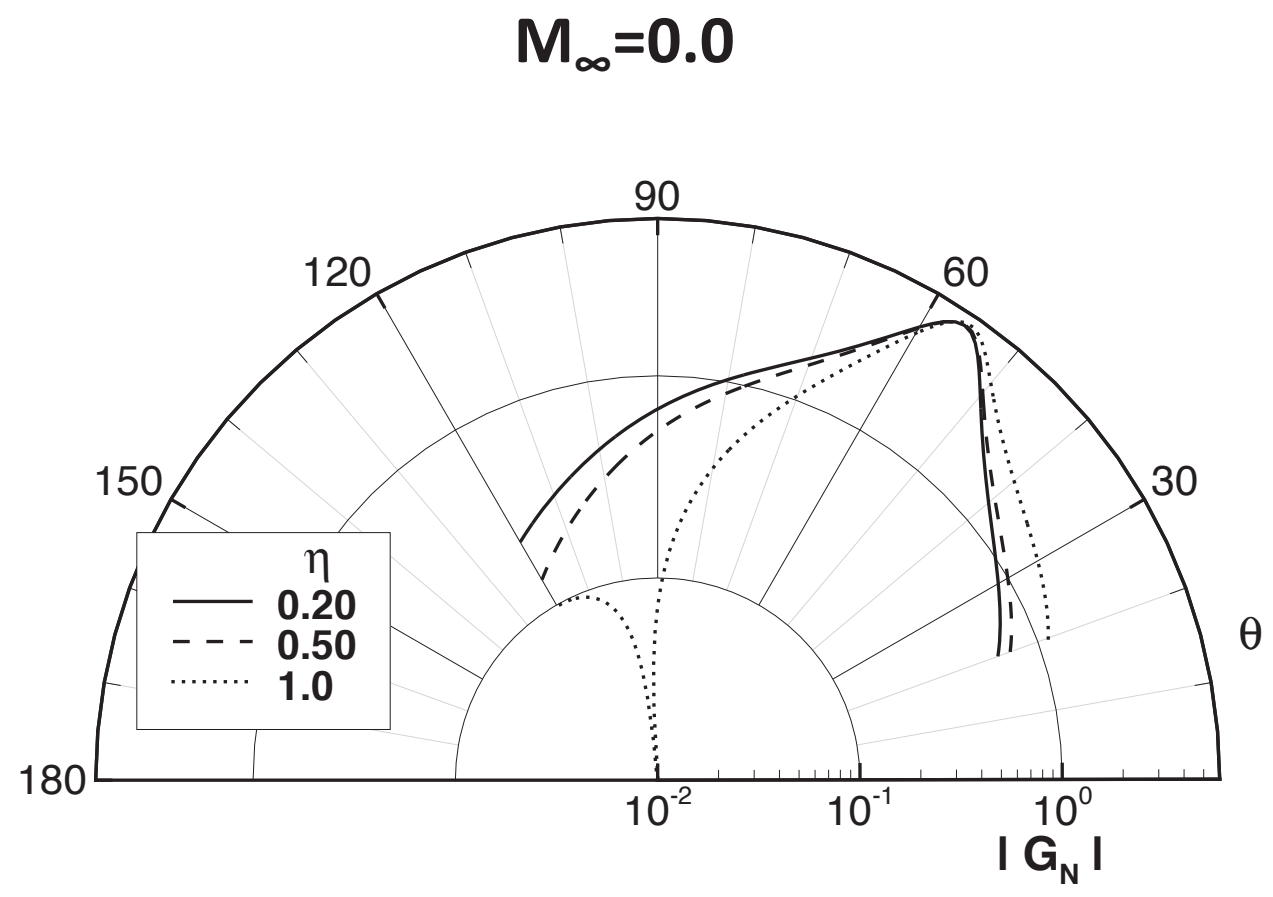

$\mathrm{St}=0.25, \mathrm{Uj}=0.90, \mathrm{TR}=10, \mathrm{Ma}=0.0, \mathrm{Z}=$ Rigid, $\phi=\pi / 2$

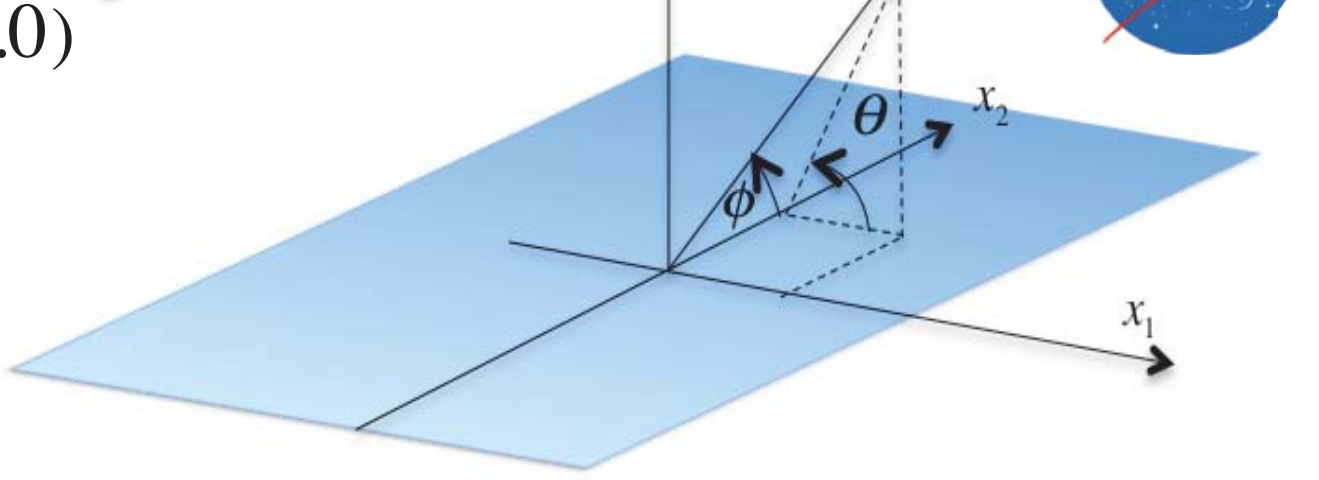

$\mathrm{M}_{\infty}=\mathbf{0 . 3 5}$

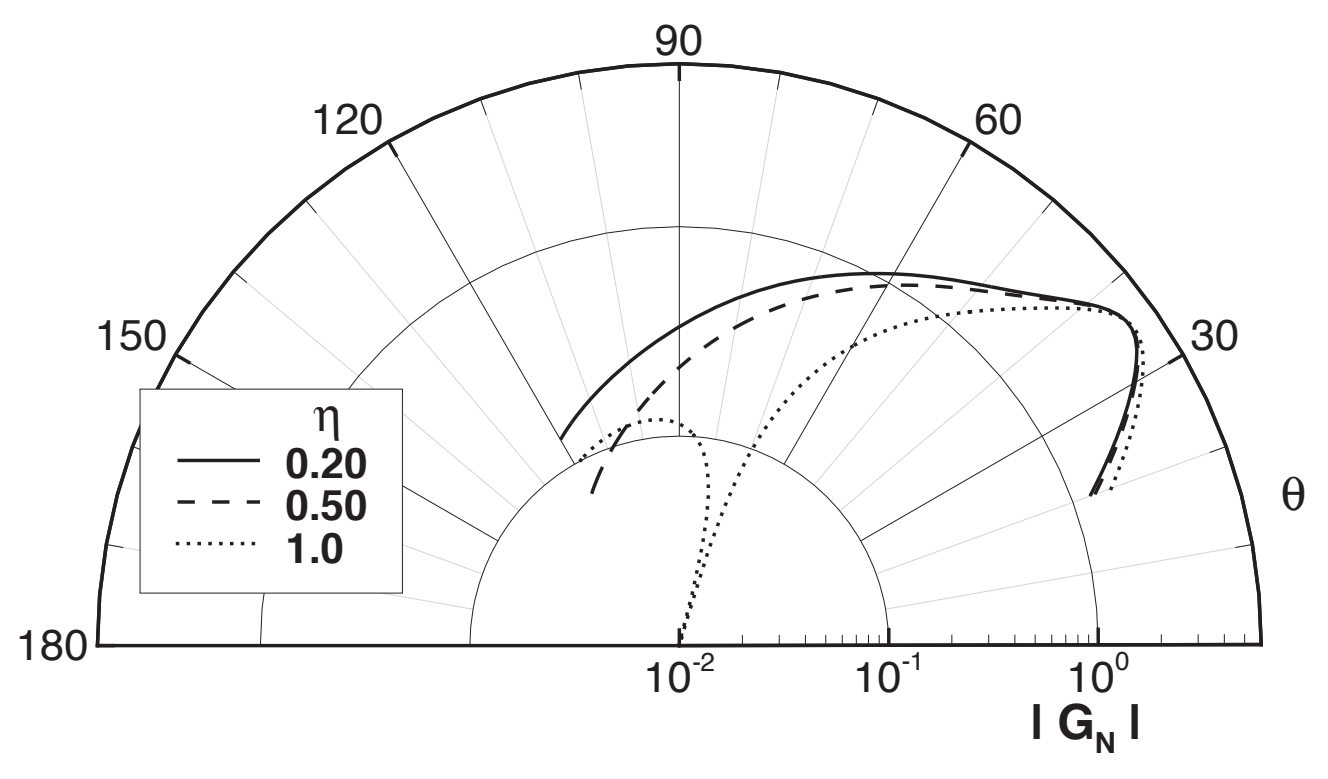

St $=0.25, \mathrm{Uj}=0.90, \mathrm{TR}=1.0, \mathrm{Ma}=0.35, \mathrm{Z}=$ Rigid, $\phi=\pi / 2$

- GF peaks at smaller down-stream angles as flight Mach number is increased 


\section{Isolated Rectangular Jet}

$V_{j}\left(x_{3}\right)=1, \quad x_{3} \rightarrow-\infty, \quad j=1,2$

$\frac{\partial V_{j}}{\partial x_{3}}-(-1)^{j} i \chi_{\infty} V_{j}=0, \quad x_{3} \rightarrow \mid \begin{array}{ll}-\infty, & j=1 \\ +\infty, & j=2\end{array}$

Jet Profile - Unheated

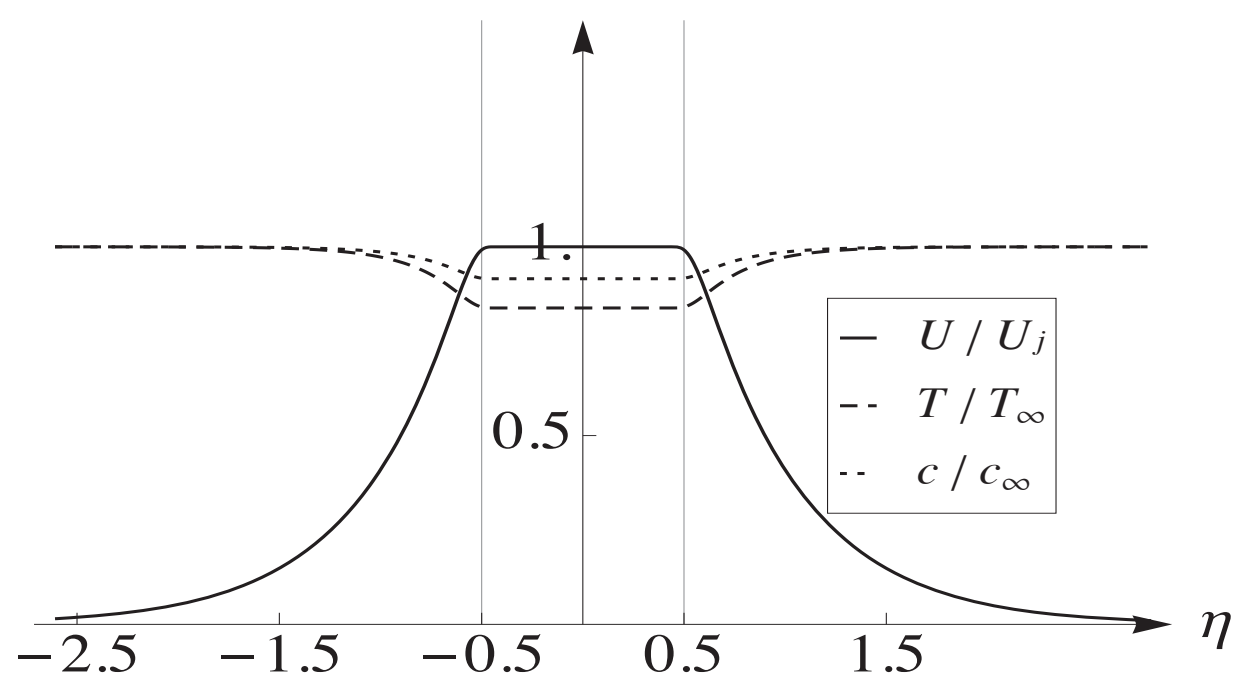

$$
\eta=y_{3} / D_{j}
$$

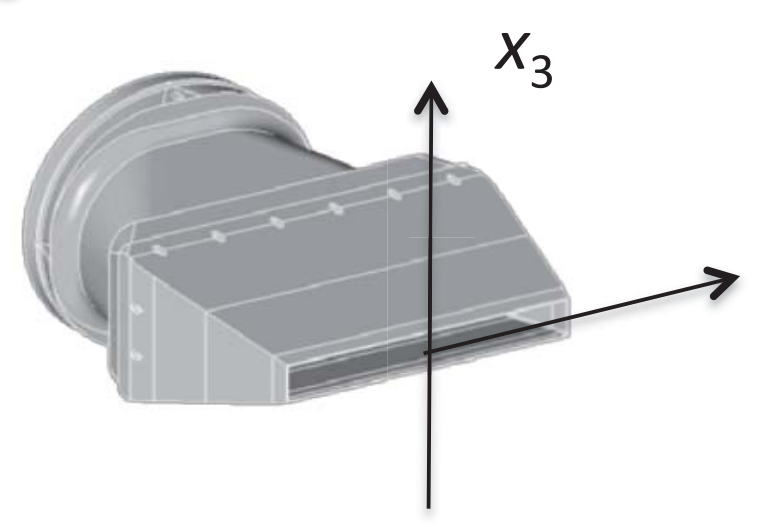

$\left|G_{N}\right| \quad$ Observer at $x_{\mathbf{3}} \rightarrow+\infty$

$\Lambda$

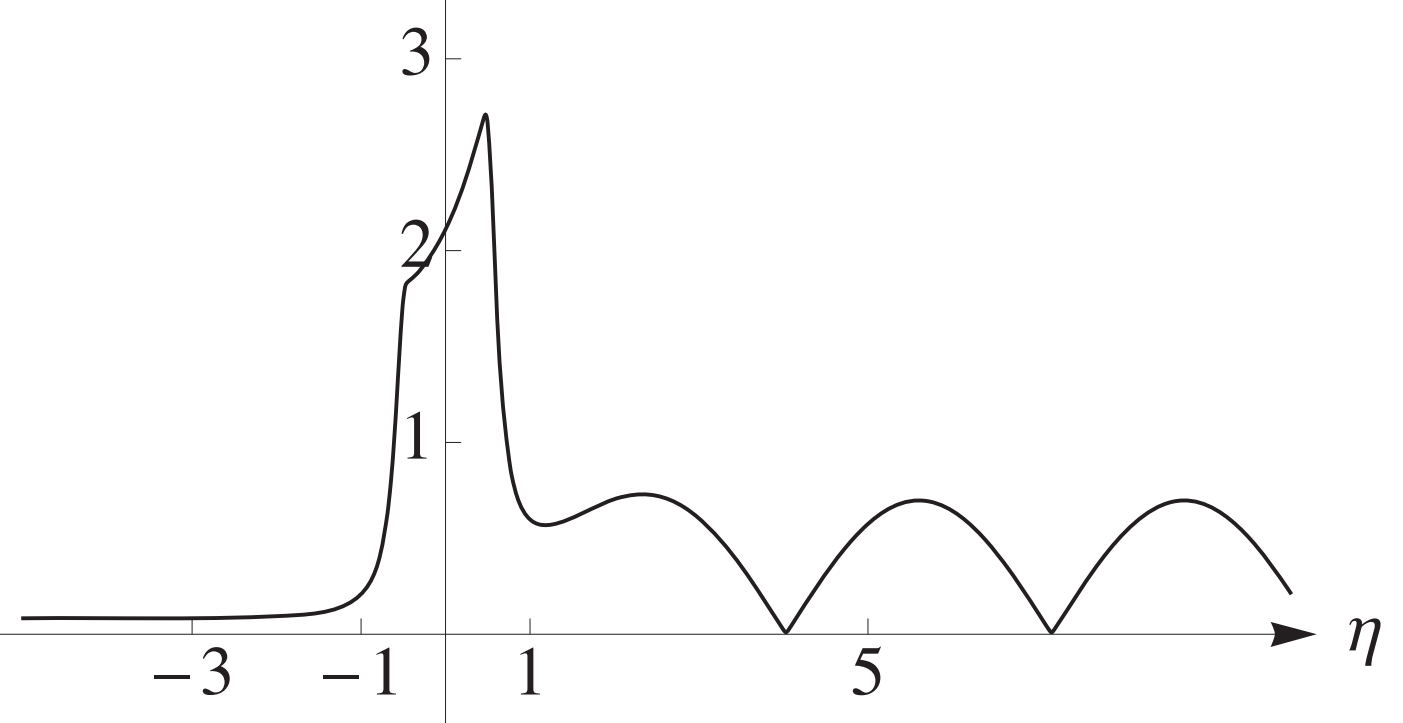

$\left(\theta=\pi / 4, S t_{o}=0.25, U_{j} / c_{\infty}=0.90, T_{R}=1.0\right)$ 


\section{Isolated vs. Reflected Jet}

$\left(M_{\mathrm{a}}=0.90, T_{\mathrm{R}}=1.0\right)$

GF Directivity $(\mathrm{St}=\mathbf{0 . 2 5})$

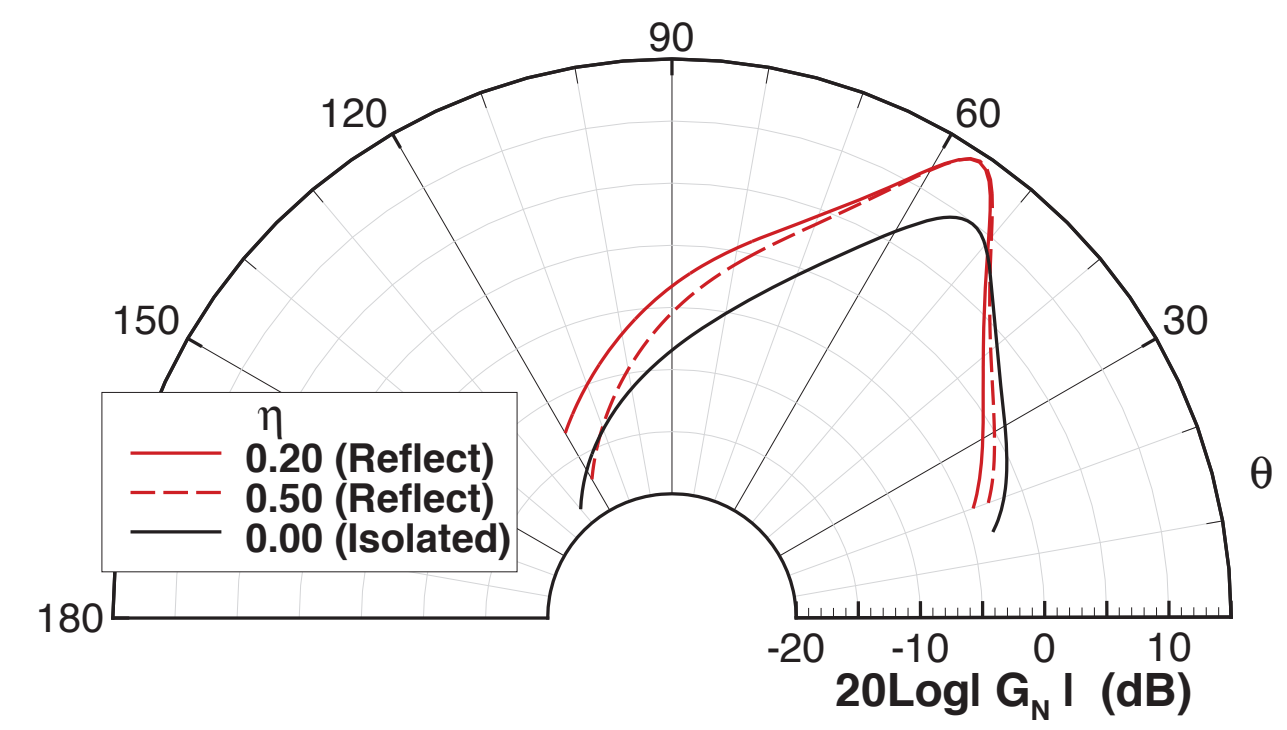

$\mathrm{St}_{\mathrm{b}}=0.25, \mathrm{Uj}=0.90, \mathrm{TR}=1.0, \mathrm{Ma}=0.0, \phi=\pi / 2$
Measured SPL $(\mathrm{St}=\mathbf{0 . 2 8}, \mathrm{AR}=\mathbf{8})$

James Bridges, 2014

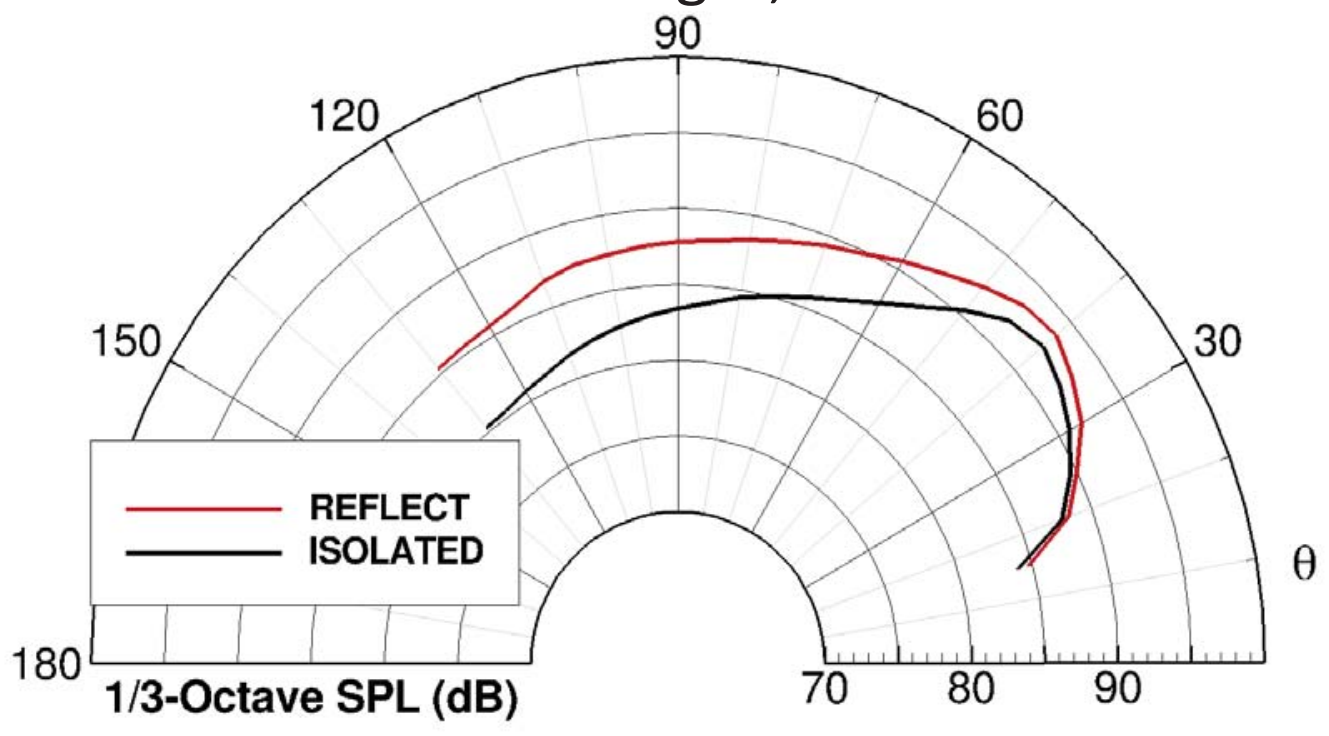

$$
X_{T E} / D_{e}=5.6, D_{e}=2.14 "
$$

- A reflecting surface enhances the GF (5-6 dB) relative to an isolated jet (at polar angles larger than peak directivity angle) 


\section{Summary}

Within the region of nonzero sources:

- GF magnitude decreases with increasing frequency

- GF magnitude decreases with increasing temperature

- At a fixed polar angle GF magnitude varies with source location, generally increases at smaller downstream angle

- Phase factor varies with source location for non-rigid surface impedance

- GF peaks at smaller down-stream angles as flight Mach number is increased

- Presence of a reflecting surface enhances the GF magnitude (5-6 dB) relative to an isolated jet at polar angles larger than peak directivity angle. 


\section{QUESTIONS ?}




\section{Measurements (JSIT Tests*)}
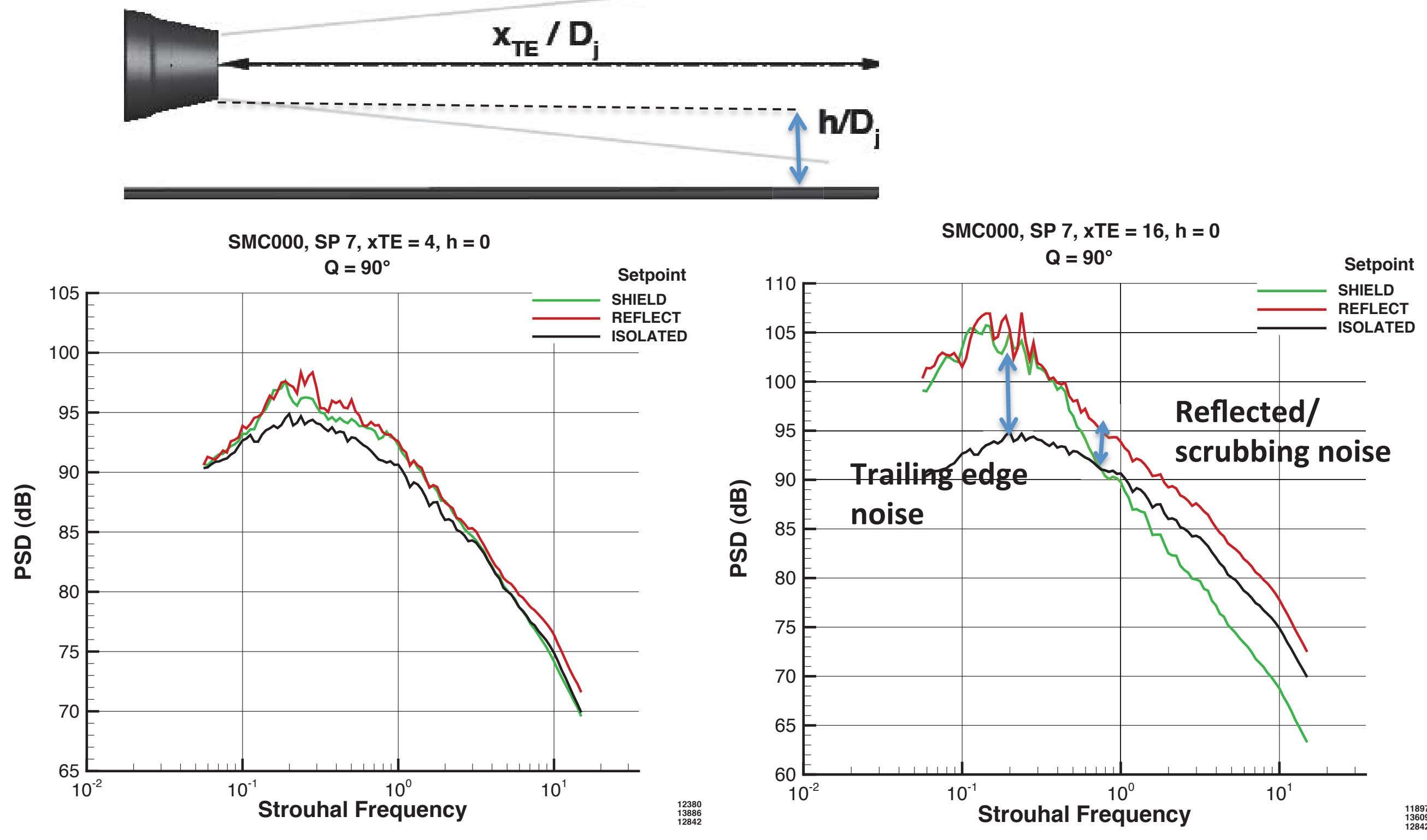

*Cliff Brown (GRC), ASME paper GT2012

* Gary Podboy (GRC), ASME paper GT2012 


\section{Mean Flow - Analytical Profiles}

- Axial Velocity $\quad \eta=y_{3} / D_{j}$

$$
\frac{U(\eta)}{U_{j}}=\mid \begin{array}{ll}
\tanh \left(\frac{D_{j} \eta}{d_{1}}\right), & \eta<1.05 \\
\frac{1}{2}\left(1+\frac{U_{\infty}}{U_{j}}\right)+\frac{1}{2}\left(1-\frac{U_{\infty}}{U_{j}}\right) \tanh \frac{1}{d_{2}}\left(\frac{1 / 2}{\eta-1}-\frac{\eta-1}{1 / 2}\right), & \eta \geq 1.05
\end{array}
$$

- Temperature

$$
\begin{aligned}
& T=T_{1}+T_{2} \\
& \frac{T_{1}\left(y_{3}\right)}{T_{\infty}}=1+\left(T_{R}-1\right) \frac{U\left(y_{3}\right)}{U_{j}}-\frac{\gamma-1}{2}\left(\frac{U\left(y_{3}\right)}{c_{\infty}}\right)^{2} \quad \text { Crocco-Busemann Law } \\
& \frac{T_{2}\left(y_{3}\right)}{T_{\infty}}=\frac{1}{d_{3}}\left(\frac{1}{2}+\frac{1}{2} \tanh \frac{1}{d_{4}}\left(\frac{1}{D_{j} \eta}-D_{j} \eta\right)\right) \quad \text { Frictional heat near the wall } \\
& D_{j}=2^{\prime \prime} \quad \delta_{o} / D_{j}=1.32 d_{1} \quad\left(d_{1}, d_{2}, d_{3}, d_{4}\right)=(0.10,2,4,3)
\end{aligned}
$$




\section{Effect of Observer Angle}

$\left(\phi=\pi / 2, S t_{o}=0.25, U_{j} / c_{\infty}=0.90, T_{R}=3.0\right)$

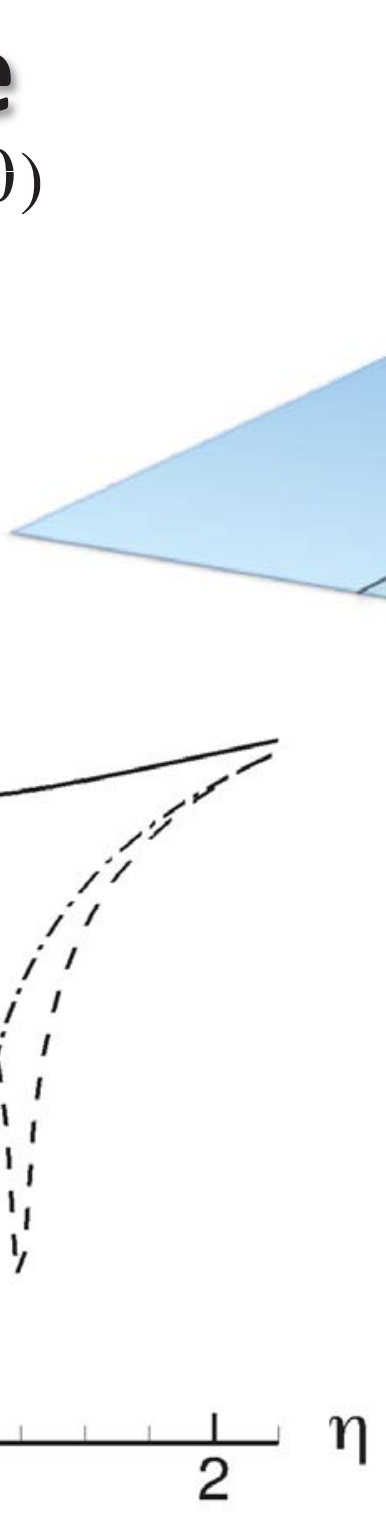

- GF amplitude varies with source location, generally increases at smaller downstream angles 